\title{
Contribution of GABAergic modulation in DRGs to electroacupuncture analgesia in incisional neck pain rats
}

\author{
Li Na Qiao',2 \\ Yong Sheng Yang ${ }^{2}$ \\ Jun Ling Liu \\ Jiang Zhu' \\ Lian Hong Tan ${ }^{2}$ \\ Yi Nan $\mathrm{Shi}^{2}$ \\ Bing Zhu ${ }^{2}$ \\ Pei Jing Rong ${ }^{2}$
}

'School of Acupuncture-Moxibustion and Tuina, Beijing University of Chinese Medicine, Beijing, China; ${ }^{2}$ Institute of Acupuncture and Moxibustion, China Academy of Chinese Medical Sciences, Beijing, China

Correspondence: Bing Zhu; Pei Jing Rong Institute of Acupuncture and

Moxibustion, China Academy of Chinese Medical Sciences, 16 Nanxiaojie Street,

Dongzhimennei, Beijing 100700, China

Tel +86 1064089409

Fax +86I06 4032682

Email zhubing@mail.cintcm.ac.cn;

drrongpj@163.com
This article was published in the following Dove Medical Press journal: Journal of Pain Research

Purpose: Acupuncture therapy is effective for relieving postoperative pain. Our previous study showed that electroacupuncture (EA) at Futu (LI18) and Hegu (LI4)-Neiguan (PC6) could alleviate incisional neck pain, which was related with its effect in upregulating $\gamma$-aminobutyric acid (GABA) expression in cervical (C3-6) dorsal root ganglions (DRGs); but whether its receptor subsets $\mathrm{GABA}_{A} \alpha 2 \mathrm{R}$ and $\mathrm{GABA}_{B} \mathrm{R} 1$ in $\mathrm{C} 3-6$ DRGs are involved in EA analgesia or not, it remains unknown.

Materials and methods: Seventy-five male Sprague Dawley rats were randomized to normal control, model, LI18, LI4-PC6, and Zusanli (ST36)-Yanglingquan (GB34) groups. The incisional neck pain model was established by making a longitudinal incision along the midline of the rats' neck, followed by repeated mechanical stimulation. EA was applied to bilateral LI18, LI4-PC6, or ST36-GB34 for 30 minutes at 4, 24, and 48 hours after operation. The thermal pain threshold of the neck was detected by a tail-flick unit, and the C3-6 DRGs were removed for assaying the immunoactivity of substance $P(S P), G A B A_{A} \alpha 2 R$, glial fibrillary acidic protein (GFAP; a marker of satellite glial cells [SGCs]), and $\mathrm{GABA}_{\mathrm{B}} \mathrm{R} 1$ and the expression of $\mathrm{GABA}_{\mathrm{A}} \alpha 2 \mathrm{R}$ and $\mathrm{GABA}_{\mathrm{B}} \mathrm{R} 1 \mathrm{mRNA}$ and proteins using immunofluorescence, real-time PCR, and Western blotting, respectively.

Results: The cervical thermal pain threshold was significantly lower in the model group than the normal group $(P<0.001)$, indicating hyperalgesia after neck incision, and was considerably increased in both EA-LI18 and LI4-PC6 groups $(P<0.001)$, but not in ST36-GB34 group compared with model group $(P>0.05)$. Immunofluorescence staining showed that $\mathrm{GABA}_{\mathrm{A}} \alpha 2$ $\mathrm{R}$ expressed on $\mathrm{SP}^{+}$neurons, and $\mathrm{GABA}_{\mathrm{B}} \mathrm{R} 1$ on SGCs. EA of LI18 and LI4-PC6 markedly suppressed the modeling-induced upregulation of the immunoactivity of SP $(P<0.001$ and $P<0.01$, respectively) and GFAP ( $P<0.01$ and $P<0.001$, respectively) and significantly reversed neck incision-induced downregulation of the expression of $\mathrm{GABA}_{A} \alpha 2 \mathrm{R}$ and $\mathrm{GABA}_{\mathrm{B}} \mathrm{R} 1 \mathrm{mRNAs}$ and proteins $(P<0.05)$.

Conclusion: EA of LI18 and LI4-PC6 has an analgesic effect in incisional neck pain rats, which is related to its effects in upregulating GABAergic inhibitory modulation on nociceptive peptidergic neurons and SGCs in cervical DRGs.

Keywords: electroacupuncture, incisional neck pain, substance P, satellite glial cells, $\mathrm{GABA}_{\mathrm{A}} \alpha 2 \mathrm{R}, \mathrm{GABA}_{\mathrm{B}} \mathrm{R} 1$

\section{Introduction}

Postoperative pain remains one of the most common challenges following surgeries, though significant accomplishments have been made over the past few decades. ${ }^{1}$ The dorsal root ganglion (DRG), a key epidural and intraspinal nerve structure that is 
responsible for primary sensory information flow from the peripheral to the spinal cord, has been demonstrated to be an important processing point of sensory signals and also a "robust target for neuromodulation therapies". Recent clinical data have shown that DRG stimulation is as effective as spinal cord stimulation in relieving various neuropathic pain syndromes including pain due to failed back surgery syndrome, complex regional pain syndromes, and chronic postsurgical pain. ${ }^{2-4}$ The analgesic effect of electrical stimulation of DRG is probably induced by interrupting the transmission of afferent impulse trains of sensory neurons as they pass through the DRG. ${ }^{5}$

Mounting evidence displayed that neuropeptides and neuropeptide receptors are known to play important roles in nociceptive signal transmission and modulation. For example, excitatory neuropeptides, substance $\mathrm{P}$ (SP) and calcitonin gene-related peptide (CGRP), were found to be expressed in small DRG neurons and SP-immunoreaction (IR) was seen in $50 \%$ of C-fiber neurons and $20 \%$ of $\mathrm{A} \delta$-fiber neurons. ${ }^{6} \mathrm{SP}$ depolarized DRG neurons and activated an inward current, thereby increasing the excitability of sensory nociceptors. ${ }^{7}$ Also, about $90 \%$ of DRG neurons were endowed with SP receptors. ${ }^{8} \gamma$-Aminobutyric acid (GABA), a traditional inhibitory neurotransmitter, binding to its receptors, exerted a tonic modulation on the nociceptive neurotransmission between primary afferents and second-order spino-thalamic tract neurons via both pre- and post-synaptic modes. ${ }^{9,10}$ Activation of GABA in DRGs resulted in a decrease in release of SP and glutamate from the primary afferent terminals. ${ }^{8}$ It was reported that majority of the DRG neurons coexpressed $\mathrm{GABA}_{\mathrm{A}}$ and SP receptors in their soma and central processes in lamina I-III of the spinal dorsal horns (DHs) ${ }^{11,12}$ and SP each other. ${ }^{13,14}$ Therefore, if SP depresses GABA-evoked response at the central terminal of primary afferent neurons and leads to a disinhibition of the "presynaptic inhibition", it would result in an enhancement of nociceptive signaling in the spinal cord..$^{15}$

In the peripheral nervous system, large, small, and medium-sized neurons located in sensory ganglia were found to be tightly surrounded by satellite glial cells (SGCs) ${ }^{16}$ that were activated in the early phase (on day 1,3 , and 7) after spinal nerve ligation operation, contributing to the occurrence of neuropathic pain. ${ }^{17}$ SGCs upregulated the production of proinflammatory cytokines such as tumor necrosis factor- $\alpha$ after peripheral nerve injury ${ }^{18}$ to increase the neuronal excitability, inducing pain. ${ }^{19,20}$ They were also found to express only $\mathrm{GABA}_{\mathrm{B}} 1 \mathrm{~b}$ receptor of the inhibitory neurotransmitter GABA. ${ }^{21}$ However, the function of $\mathrm{GABA}_{\mathrm{B}} 1 \mathrm{~b}$ subunit expressed on SGCs in incisional pain processing tasks is not clear.

Pain is particularly sensitive to acupuncture. ${ }^{22}$ It was reported that electroacupuncture (EA) treatment can increase the pain threshold (PT) by decreasing the expression of $\mathrm{P} 2 \mathrm{X}(3)$ receptors in DRG neurons in chronic constriction injury rats and by attenuating ATP and $\alpha, \beta$-meATP-evoked currents. ${ }^{23}$ EA may affect the progression of experimental inflammatory pain by modulating the expression of $N$-methylD-aspartic acid receptors in primary sensory neurons, in particular, IB4-positive small neurons. ${ }^{24}$

Our previous studies demonstrated that EA intervention relieved thermal hyperalgesia in incisional neck pain rats via its effects in downregulating the expression of SP/ CGRP, connexin 43 (Cx43), reducing the activity of SGCs and release of proinflammatory cytokines, and upregulating the inhibitory transmitter GABA in the primary sensory neurons of cervical DRGs. ${ }^{25,26}$ However, the mechanism by which EA relieves incisional neck pain by regulating the activities of neurons and satellite glial cells remains unclear. Also, whether GABAergic modulation on nociceptive SP neurons and SGCs in the cervical DRGs contribute to EA analgesia in the same pain model or not has not been reported up to now. Thus, the present study was designed to explore if the GABA receptor subunits are implicated in EA-induced pain relief in incisional neck pain rats, so as to better our understanding about the mechanism of acupuncture therapy for post-thyroidectomy pain.

\section{Materials and methods}

\section{Animals}

Seventy-five adult male Sprague Dawley rats (8 weeks in age; $200-220 \mathrm{~g}$ ) were purchased from Beijing Union Medical College, and housed under standard laboratory conditions (12 hours alternate light-dark cycle) and given free access to standard chow pellet diet and water. The experimental protocols were approved by the ethics committee of the Institute of Acupuncture-moxibustion, China Academy of Chinese Medical Sciences (reference no. 20140014) and conformed to the Guidelines for Laboratory Animal Care and Use of Chinese Ministry of Science and Technology (2006) and the National Institutes of Health Guide for Care and Use of Laboratory Animals (1996). In addition, efforts were made to minimize the number of animals used for the experiments and their sufferings. The rats were randomly assigned to five groups ( $\mathrm{n}=15$ in each): normal, model, LI18, LI4-PC6, and ST36-GB34. Rats in the normal group received only isoflurane anesthesia, 
those in the model group underwent neck incision under isoflurane anesthesia, and those in the LI18, LI4-PC6, and ST36-GB34 groups received EA (at these respective points or point combinations) plus neck incision under isoflurane anesthesia. The protocol used in this study is shown in Figure 1.

\section{Establishment of incisional neck pain model}

The incisional neck pain model was established by making a $2.0 \mathrm{~cm}$ longitudinal incision along the midline of the neck under isoflurane ( $1 \%-2 \%$ in oxygen) delivered via an anesthesia unit (Midmark Animal Health, Versailles, OH, USA), followed by repeated blunt dissection stimulation of the bilateral sternohyoideus muscles in the thyroid gland region for about 10 minutes using a pair of forceps. The incision was then sutured in layers with 4.0 surgical silk braided suture at an interval of about $0.5 \mathrm{~cm}$.

\section{Measurement of thermal PT}

The thermal PT of the neck incision region was measured at baseline and 48 hours after surgical incision using a tailflick unit (37360; UGO Basile, Gemonio VA, Italy). ${ }^{25,27}$ The heat intensity was set to 50 units, with a cutoff time of 30 seconds to avoid tissue damage. The neck withdrawal latency was detected three times for each rat at an interval of about 5 minutes, and the average value was used to assess the pain severity. The researcher who performed thermal PT measurements was blind to group assignment and did not participate in the acupuncture procedure.

\section{EA intervention}

Under light anesthesia with $1.5 \%$ isoflurane, the rats of the three EA groups underwent EA stimulation following insertion of acupuncture needles $(0.25 \times 25 \mathrm{~mm}$; Huatuo, Suzhou Medical Co. Ltd., Jiangsu, China) into bilateral LI18, LI4 and PC6, or ST36 and GB34 at a depth of about 2-3 mm, respectively. In humans, LI18 is located at the posterior border of sternocleidomastoid, 3 cun (about $6 \mathrm{~cm}$ ) lateral to the laryngeal prominence. In rats, the equivalent location is about $1 \mathrm{~cm}$ lateral to the thyroid cartilage. The remaining points were located according to an atlas of experimental animal acupuncture points, ${ }^{28}$ that is, LI4 located between the first and second metacarpal bones, PC6 about $3 \mathrm{~mm}$ to the transverse stripe of the wrist at the axopetal end, ST36 about $5 \mathrm{~mm}$ inferior to the capitulum fibulae and posterolateral to the hind limb knee joint, and GB34 about $5 \mathrm{~mm}$ superolateral to ST36. After insertion, the needle handles were connected to a HANS-200A Analgesia Apparatus (Nanjing Jisheng Medical Technology Co. Ltd., Jiangsu, China) and this was followed by turning on the apparatus to stimulate the abovementioned acupoints for 30 minutes every time, at an alternating frequency of $2 / 100 \mathrm{~Hz}$ and an intensity of $1 \mathrm{~mA}$. The treatment was conducted at 4, 24, and 48 hours after modeling. Animals of the model group underwent the same anesthetic procedures, but without EA stimulation.

\section{Necropsy and tissue preparation}

Following EA treatment and thermal PT measurement, those rats $(n=25)$ were deeply anesthetized with $20 \%$ urethane and transcardially perfused through the ascending aorta with

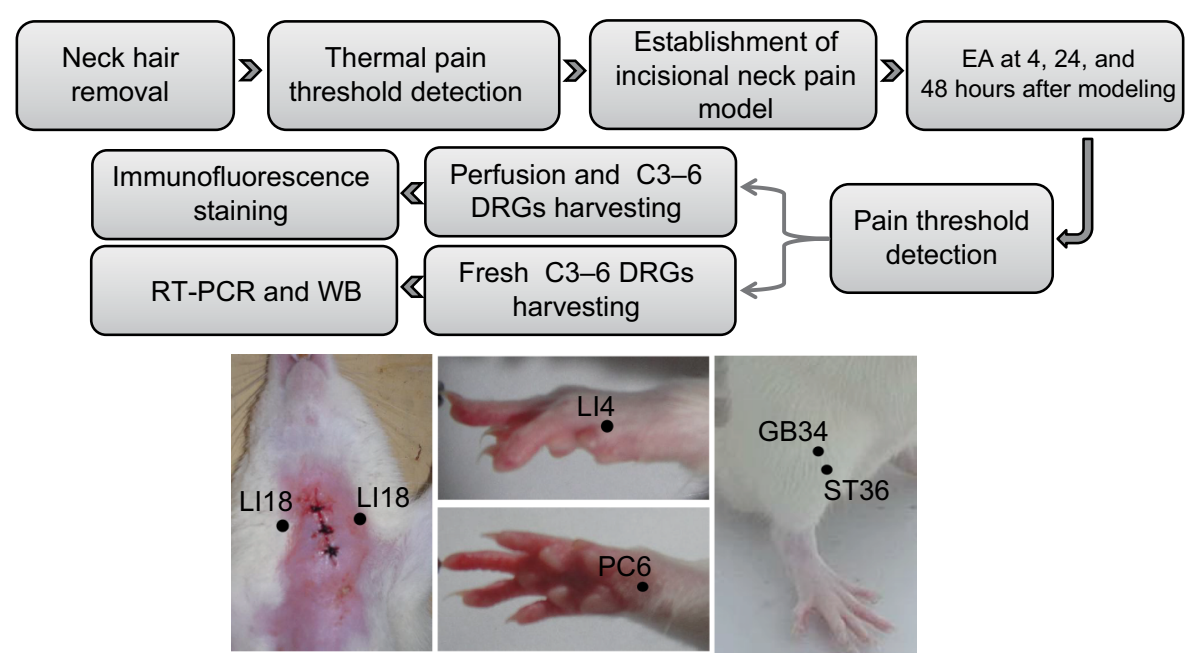

Figure I Summary of the protocol and the location of acupoints used in this study.

Abbreviations: DRG, dorsal root ganglion; EA, electroacupuncture; RT-PCR, real time PCR; WB, Western Blot. 
normal saline $(400 \mathrm{~mL})$, followed by $4 \%$ paraformaldehyde in $0.1 \mathrm{M}$ PBS (pH 7.4, $400 \mathrm{~mL}$ ). Then, the C3-C6 DRGs were carefully removed to be kept in $0.1 \mathrm{M}$ PBS containing $30 \%$ sucrose at $4{ }^{\circ} \mathrm{C}$ for subsequent immunofluorescence testing. DRGs from the remaining 50 rats ( $n=10$ per group) were removed on an ice plate rapidly frozen in liquid nitrogen and stored at $-80^{\circ} \mathrm{C}$ for RNA and protein extraction later.

\section{Immunofluorescence assay}

The DRG samples were sectioned to a thickness of $30 \mu \mathrm{m}$ by a freezing microtome (Thermo Fisher Scientific, Waltham, MA, USA) and maintained at room temperature for 1 hour. Sections were blocked with 5\% donkey serum for 30 minutes and incubated with $\mathrm{GABA}_{\mathrm{A}} \alpha 2 \mathrm{R}$ (AGA-002; Alomone Labs, Jerusalem, Israel) and SP (sc58591; Santa Cruz Biotechnology Inc., Dallas, TX, USA), or GABA R1 (ab90883; Abcam, Cambridge, MA, USA) and GFAP (a marker for SGCs activities, 3670S; Cell Signaling) at $4{ }^{\circ} \mathrm{C}$ overnight, washed three times with $0.1 \mathrm{M}$ PBS, and then incubated in donkey anti-mouse IgG conjugated Alexa Fluor488 (Thermo Fisher Scientific) and donkey anti-rabbit IgG conjugated Alexa Fluor594 (Thermo Fisher Scientific) for 2 hours under room temperature. Control immunostaining was performed by substituting the primary antibody with normal serum.

$\mathrm{SP}, \mathrm{GABA}_{\mathrm{A}} \alpha 2 \mathrm{R}, \mathrm{GFAP}$, and $\mathrm{GABA}_{\mathrm{B}} \mathrm{R} 1$ positive immunoactivity in the bilateral DRGs (C3-C6) was detected from every three randomly selected sections of each DRG by a technician who was blind to the grouping and calculated their average optical intensity using an optical imaging analysis system (DXM1200c; Nikon Corporation, Tokyo, Japan). Some representative section photos were taken using a laser scanning confocal microscope (FV1000; Olympus Corporation, Tokyo, Japan). Digital images were then processed with Adobe Photoshop CS2 (Adobe Systems, San Jose, CA, USA).

\section{Quantitative real-time PCR}

In the subset of 25 rats, total RNA was extracted with Trizol (CW0581; Beijing ComWin Biotech Co., Ltd, Beijing, China) and then reverse transcribed into cDNA with a cDNA Synthesis Kit (CW0744; Beijing ComWin Biotech Co., Ltd, Beijing, China). Gene expression levels were measured by quantitative real-time PCR (LightCycler480; Roche, Switzerland) using the following primer sequences: $\mathrm{GABA}_{\mathrm{A}} \alpha 2 \mathrm{R}$, forward: 5'-TTACTTCACGAAAAGAGGATGGGC-3' and reverse: 5'-AAAGATTCGGGGCGTAGTTGG-3'; GAB$A_{B}$ R1 forward: 5'-TGGCACTGGCTGCTGTCTTCC-3' and reverse: 5'-TAGAGCCATAGCCCAGACTAAAGCC-3'; and GAPDH forward: 5'-CCTTCCGTGTTCCTACCCC-3' and reverse: 5'-GCCCAGGATGCCCTTTAGTG-3'.
Each reaction mixture consisted of $2 \mu \mathrm{L}$ of cDNA, 10 $\mu \mathrm{L}$ REAL SYBR Mixture $(2 \times), 0.8 \mu \mathrm{L}(10 \mu \mathrm{mol} / \mu \mathrm{L})$ both forward and reverse primers, and $7.2 \mu \mathrm{L}$ PCR-grade water, equating to a final volume of $20 \mu \mathrm{L}$. PCR was performed under the following conditions: 30 seconds at $95^{\circ} \mathrm{C}$, followed by 45 cycles of 5 seconds at $95^{\circ} \mathrm{C}$ and 40 seconds at $60^{\circ} \mathrm{C}$. The relative expression was calculated in accordance with the $\Delta \Delta \mathrm{Ct}$ method. Relative mRNA levels were expressed as $2^{-\Delta \Delta \mathrm{Ct}}$ values. The researcher evaluating mRNA expression was blind to group assignment and not involved in the acupuncture procedure.

\section{Western blotting}

The frozen C3-6 DRG samples of 25 rats were prepared after tissue lysis and centrifugation for SDS-polyacrylamide gel electrophoresis. The protein with SDS sample buffer was mixed with $\beta$-mercaptoethanol, separated under reducing conditions on a $5 \%$ SDS polyacrylamide gel, and finally transferred to a nitrocellulose membrane. Blocking was carried out in 3\% BSA (VWR International LLC, Solon, $\mathrm{OH}, \mathrm{USA}$ ) solution for 30 minutes at room temperature. The membrane was incubated with rabbit anti-GABA $\alpha 2 R$ (1:1,000, AGA-002; Alomone Labs) and rabbit anti-GAB$A_{B} R 1$ (1:1,000, ab90883; Abcam). GAPDH (1:20,000; YM3029, ImmunoWay, Plano, TX, USA) was used as a standard. After overnight agitation at $4^{\circ} \mathrm{C}$, the sample was then incubated with HRP-conjugated goat anti-rabbit antibody (1:10,000; Jackson ImmunoResearch Laboratories, West Grove, PA, USA) for 1 hour at room temperature. The bands were acquired by enhanced chemiluminescence (GE Healthcare Biosciences, Piscataway, NJ, USA), and the blots were scanned and quantified using TotalLab Quant analysis software (TotalLab Limited, Newcastle Upon Tyne, England) and the result was expressed as the ratio of target gene immunoreactivity to GAPDH immunoreactivity.

\section{Statistical analysis}

Data were analyzed with the SPSS version 16.0 (SPSS Inc., Chicago, IL, USA). Data were expressed as mean \pm SD and were analyzed by one-way ANOVA, followed by least significant difference (LSD) test for multiple comparisons between groups. $P<0.05$ was considered to be statistically significant.

\section{Results Effect of EA on thermal PT}

As shown in Figure 2, at 48 hours after neck incision, the thermal PT was significantly lower in the model group than in the normal group $(12.95 \pm 1.92$ vs $19.23 \pm 0.92$ seconds, one-way ANOVA, LSD, $P=0.000, \mathrm{n}=15)$. After EA, relevant 


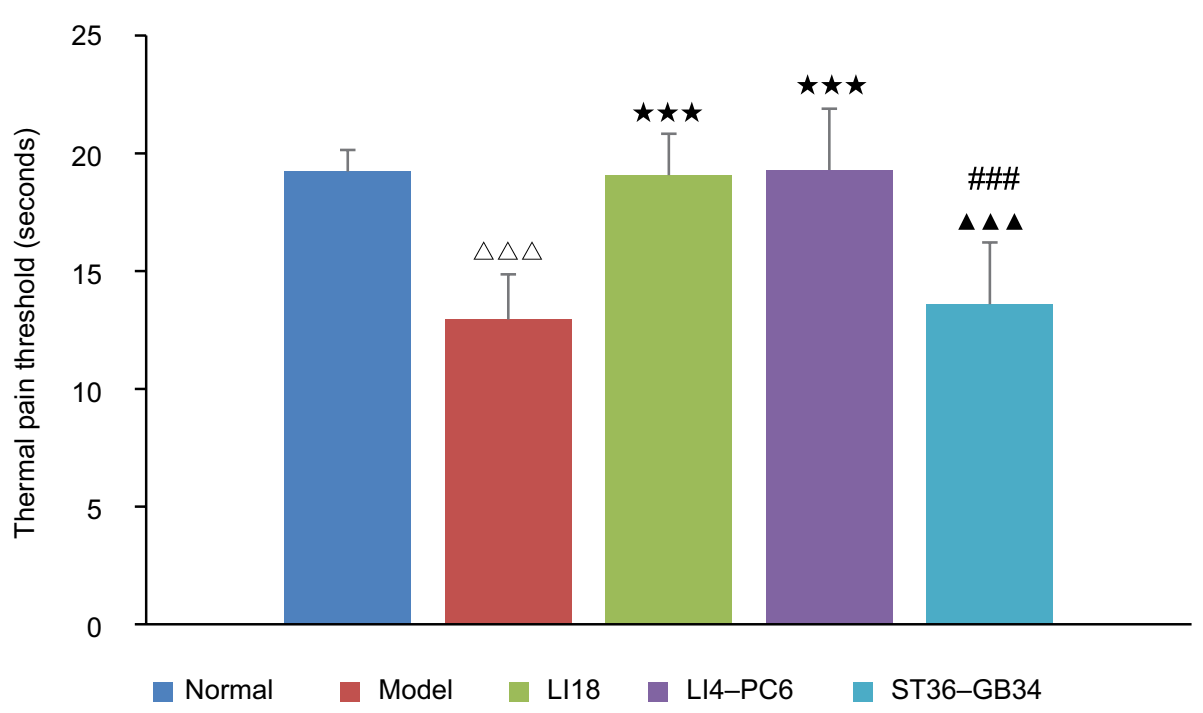

Figure 2 Comparison of thermal pain threshold among the normal, model, LII8, LI4-PC6, and ST36-GB34 groups ( $\mathrm{n}=15$ per group).

Notes: One-way ANOVA, LSD $t$-test. Data are presented as mean \pm SD of the mean. ${ }^{\Delta \triangle} P<0.00$ I vs the normal group. ${ }^{* * * P}<0.00 \mathrm{I}$ vs the model group. ${ }^{\Delta} \wedge{ }^{\wedge} P<0.00 \mathrm{I}$ vs the LII 8 group. $P<0.00$ I vs the LI4-PC6 group.

Abbreviation: LSD, least significant difference.

to the model group, the thermal PT values were considerably higher in the LI18 and LI4-PC6 groups (19.07 \pm 1.76 vs $12.95 \pm 1.92$ seconds; $19.29 \pm 2.60$ vs $12.95 \pm 1.92$ seconds, one-way ANOVA, LSD, both $P=0.000, \mathrm{n}=15)$, but not in the

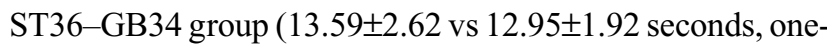
way ANOVA, LSD, $P=0.6254$. $\mathrm{n}=15, F=12.465$, degrees of freedom $[d f]=14)$.

\section{Effect of EA on SP and GABA $A_{A} R$ immunoreactivity}

Immunofluorescence staining showed that SP-IR positive substances were granular in appearance and distributed throughout the cytoplasm. The SP-IR positive nerve fibers were also found between neuronal cell bodies. The diameters of SP-IR positive neurons ranged from 8 to $27 \mu \mathrm{m}$, being in the range of small- sized cells (diameter $<27 \mu \mathrm{m}$; Figure $3 \mathrm{~A}$ ). The $\mathrm{GABA}_{\mathrm{A}} \mathrm{R}$-labeled $\mathrm{DRG}$ neurons were in the range of small-sized $(<30 \mu \mathrm{m})$ and medium-sized (31-40 $\mu \mathrm{m})$ cells (Figure 3A). The mean intensity of SP-IR positive neurons was notably greater $(P=0.000, F=11.324$, $d f=4)$, and $\mathrm{GABA}_{\mathrm{A}} \alpha 2 \mathrm{R}$ immunoactivity was significantly decreased $(P=0.000, F=27.273, d f=4)$ in the model group relative to the normal group at 48 hours after operation. After EA, compared with the model group, the SP immunoactivity was markedly downregulated and the $\mathrm{GABA}_{\mathrm{A}} \alpha 2 \mathrm{R}$ immunoactivity was considerably upregulated in both the LI1 8 group $(P=0.000, P=0.000)$ and the LI4-PC6 group
$(P=0.006, P=0,000)$, but not in the ST36-GB34 group $(P=0.688, P=0.450$; Figure $3 \mathrm{~B})$.

\section{Effect of EA on GFAP and GABA ${ }_{B} R$ immunoreactivity}

After modeling, the immunoactivity of GFAP was markedly increased $(P=0.000, F=10.101, d f=4)$ and $\mathrm{GABA}_{\mathrm{B}} \mathrm{R} 1$ immunoactivity was significantly decreased $(P=0.002$, $F=5.782, d f=4)$. After EA, the immunoactivity of GFAP was considerably downregulated and that of $\mathrm{GABA}_{\mathrm{B}} \mathrm{R} 1$ remarkably upregulated in both LI18 group ( $P=0.006$ and $P=0.027$, respectively) and LI4-PC6 group $(P=0.000$ and $P=0.047$, respectively), but not in the ST36-GB34 group $(P=0.39$ and $P=0.530$, respectively), compared with the model group (Figure 4A, B).

\section{Effect of EA on GABA $A_{A} \alpha 2 R$ mRNA and protein expression}

To further confirm the involvement of $\mathrm{GABA}_{\mathrm{A}} \alpha 2 \mathrm{R}$ modulation in the analgesic effect of EA, we continued to analyze the expression of $\mathrm{GABA}_{\mathrm{A}} \alpha 2 \mathrm{R}$ gene and protein in C3-6 DRGs. The results of real-time PCR and Western blot showed that compared with the normal group, the expression levels of $\mathrm{GABA}_{\mathrm{A}} \alpha 2 \mathrm{R}$ mRNA and protein were significantly decreased in the model group $(P=0.033, F=5.252, d f=4$ and $P=0.0015$, $F=6.121, d f=4$, respectively; Figure $5 \mathrm{~A}$ and B). After EA intervention, the decreased expression of $\mathrm{GABA}_{\mathrm{A}} \alpha 2 \mathrm{R}$ 
A

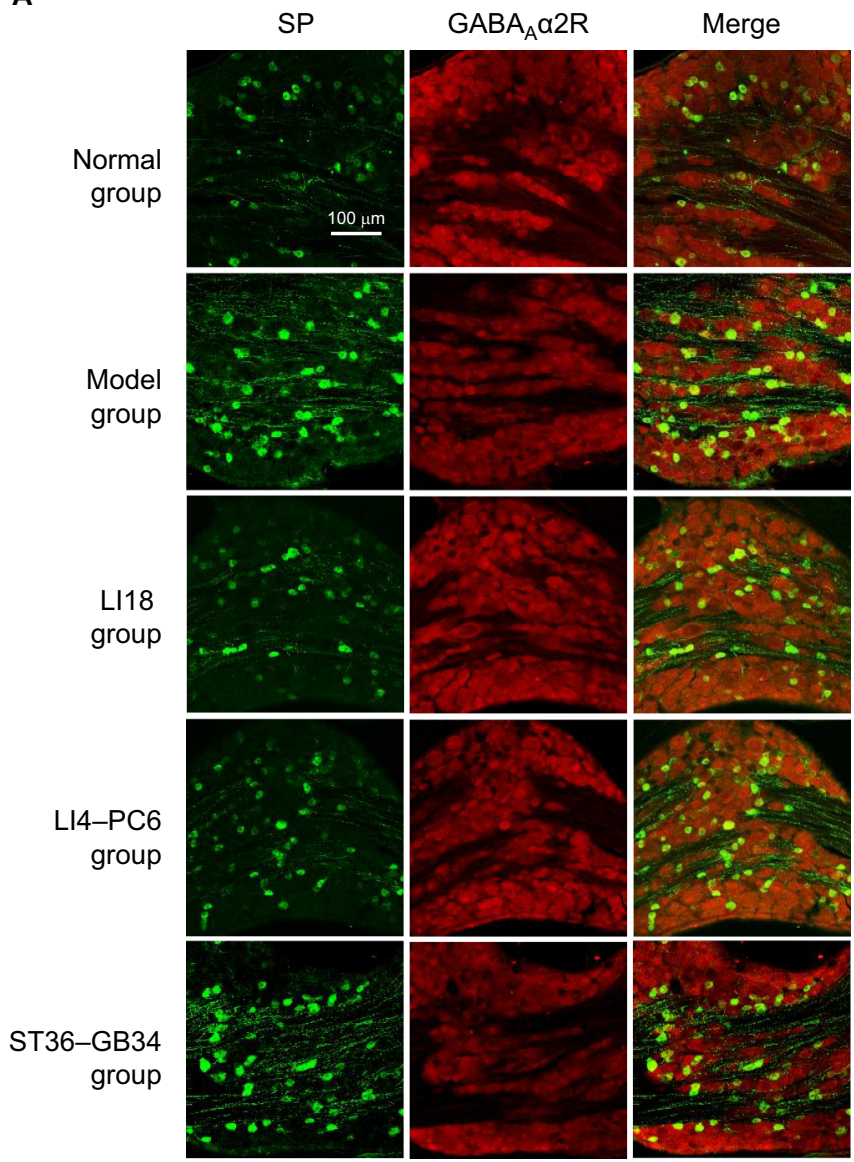

B
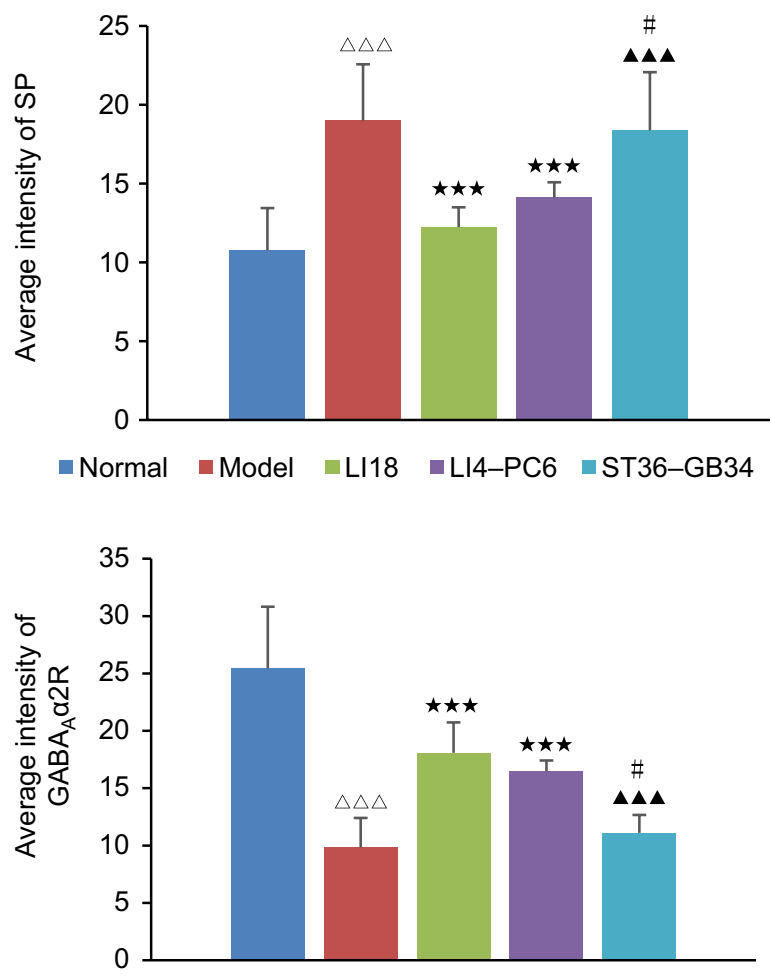

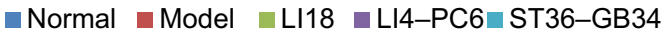

Figure 3 Effect of EA on immunoactivity of SP and GABA $\alpha 2 R$ in the cervical C3-6 DRGs in neck incisional pain rats.

Notes: (A) Representative images of SP (green) and GABA $A 2 R$ (red) expressions in the C3-6 DRGs. GABA $A 2 R$ was expressed on SP-positive neurons. (B) Average immunofluorescence intensity of SP and GABA $\alpha 2 R$ in C3-6 DRGs. The immunoactivity of SP and GABA $\alpha 2 R$ was increased and decreased, respectively, after incisional operation compared with normal rats, and after EA at LII 8 and LI4-PC6, the immunoactivity of SP was downregulated and of GABA $\alpha 2 R$ upregulated compared with the model group ( $n=5$ per group). Data were analyzed using one-way ANOVA and expressed as mean \pm SD of the mean. ${ }^{\Delta \Delta} \Delta P<0.00 \mathrm{I}$ vs the normal group, ${ }^{* * *} P<0.00 \mathrm{I}$ vs the model group, ${ }^{\triangle \triangle}{ }^{\wedge}<0.001$ vs the LII 8 group, ${ }^{\# P}<0.05$ vs the LI4-PC6 group.

Abbreviations: DRG, dorsal root ganglion; EA, electroacupuncture; GABA, $\gamma$-aminobutyric acid; SP, substance $P$.

mRNA and protein was obviously upregulated in both LI18 ( $P=0.041$ and $P=0.003$, respectively) and LI4-PC6 groups ( $P=0.000$ and $P=0.000$, respectively), but not in the ST36GB34 group ( $P=0.600$ and $P=0.316$, respectively) relative to the model group.

\section{Effect of EA on GABA $R I$ mRNA and protein expression}

As shown in Figure 6A and B, the results of real-time PCR and Western blotting indicated that compared with the normal group, the expression of $\mathrm{GABA}_{\mathrm{B}} \mathrm{R} 1 \mathrm{mRNA}$ and protein was significantly downregulated in the model group $(P=0.000$, $F=18.022, d f=4$ and $P=0.0015, F=5.161, d f=4$, respectively). Following EA, the decreased expression of $\mathrm{GABA}_{\mathrm{B}} \mathrm{R} 1 \mathrm{mRNA}$ and protein was notably increased in both $\operatorname{LI} 18$ ( $P=0.000$ and $P=0.002$, respectively $)$ and LI4-PC6 $(P=0.011$ and $P=0.004$, respectively) groups, rather than in the ST36-GB34 group ( $P=0.11$ and $P=0.31$, respectively) in comparison with the model group (Figure 6).

\section{Discussion}

To our knowledge, the present study has for the first time investigated the role of DRGs' GABAergic modulation in acupuncture analgesic effect in incisional neck pain rats. Our results demonstrated that thermal hyperalgesia of the incisional neck region was significantly reduced by EA of LI18 and LI4-PC6, the same and neighboring neurosegmental stimulation, respectively, rather than by EA of ST36-GB34, the distant neurosegmental stimulation. After EA of LI18 and LI4-PC6, the neck incision-induced increase in immunoactivity of SP and GFAP as well as decrease in the expression of $\mathrm{GABA}_{\mathrm{A}} \alpha 2 \mathrm{R}$ and $\mathrm{GABA}_{\mathrm{B}} \mathrm{R} 1 \mathrm{mRNA}$ and proteins was 
A

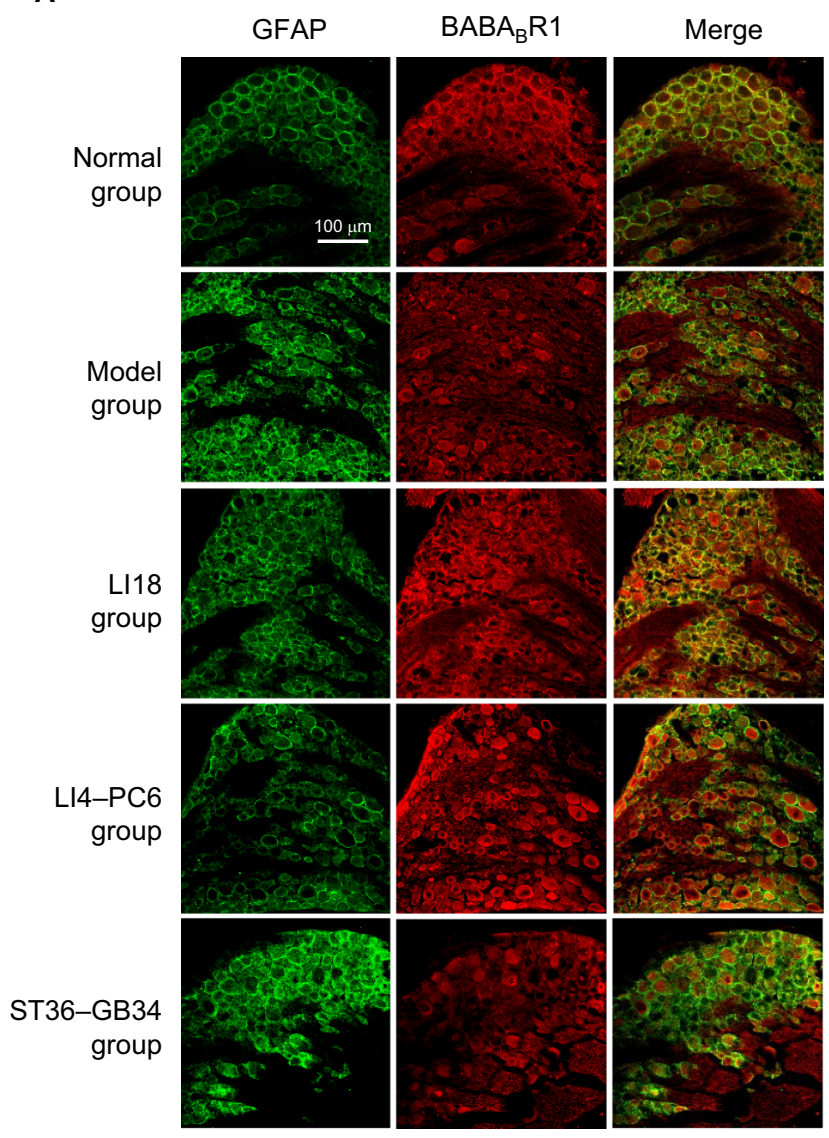

B

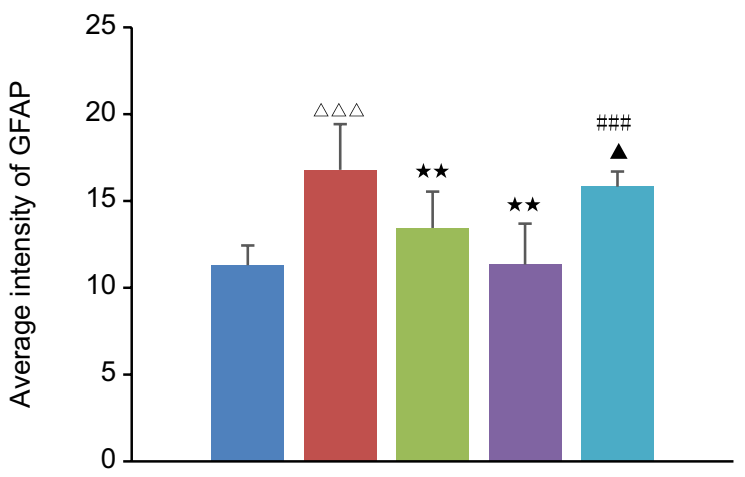

Normal $n$ Model $n$ LI18 $n$ LI4-PC6 $\backsim$ ST36-GB34

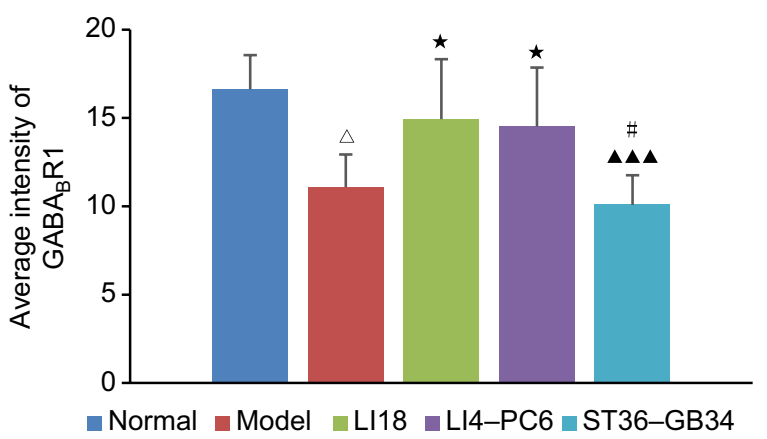

Figure 4 Effect of EA on the immunofluorescence intensity of GFAP and GABA ${ }_{B} R I$ in C3-6 DRGs in neck incisional pain rats.

Notes: (A) Representative images of GFAP (green) and GABA $R I$ (red) expressions in C3-6 DRGs, and GABA ${ }_{B} R I$ was expressed on SGCs. (B) Average immunofluorescence intensity of GFAP and GABA $R I$ in C3-6 DRGs. The GFAP and GABA ${ }_{B} R I$ immunoactivity in the model group was increased and decreased, respectively, compared with normal rats, and after EA at LII8 and LI4-PC6, the increased GFAP immunolabeling and the decreased GABA $R$ RI immunolabeling in C3-6 DRGs induced by cervical incision were reversed $(n=5$ per group). Data were analyzed using one-way ANOVA and expressed as mean $\pm S D$ of the mean. $\triangle P<0.05, \Delta \triangle P<0.001$ vs the normal group; $* P<0.05$, ${ }^{*} * P<0.0$ I vs the model group; ${ }^{\wedge} P<0.05,{ }^{\wedge}{ }^{\wedge} P<0.001$ vs the LII 8 group; ${ }^{*} P<0.05$, ${ }^{\prime \prime} P<0.001$ vs the LI4-PC6 group.

Abbreviations: DRG, dorsal root ganglion; EA, electroacupuncture; GABA, $\gamma$-aminobutyric acid; GFAP, glial fibrillary acid protein; SGCs, satellite glial cells.

considerably reversed. In contrast, EA at ST36-GB34 did not induce significant change in the immunoactivity of SP and GFAP and the expression of $\mathrm{GABA}_{\mathrm{A}} \alpha 2$ and $\mathrm{GABA}_{\mathrm{B}} \mathrm{R} 1$ mRNA and protein. It showed that EA intervention-induced pain relief in incisional neck pain rats may be achieved by way of segmental nerve reflex and upregulation of $\mathrm{GABA}_{\mathrm{A}} \alpha 2 \mathrm{R}$ and $\mathrm{GABA}_{\mathrm{B}} \mathrm{R} 1$ and downregulation of SP and SGCs activity in the same and neighboring neurosegmental DRGs.

It was reported that the spinal GABA(B) mechanism was involved in the intensity of analgesic effect of EA at $100 \mathrm{~Hz}$ and spinal GABA(A) was involved in the duration of the effect in rats, ${ }^{29}$ indicating that the analgesic effect of EA depends at least partially on the GABAergic system.

Several previous studies demonstrated that nonsynaptically released diffusible chemical messengers, such as $\mathrm{SP},{ }^{30}$ CGRP ${ }^{31}$ glutamate, ${ }^{32}$ ATP, ${ }^{33}$ and GABA,${ }^{34}$ may modify the somatic excitability of neurons in the sensory ganglia. SP, a neuropeptide, is involved in the transmission and modulation of nociceptive information in the nervous system. ${ }^{35}$ GABA, the main inhibitory transmitter in the central nervous system, modulates neurotransmitter release and neuronal excitability by acting on two types of specific receptors as $\mathrm{GABA}_{\mathrm{A}} \mathrm{Rs}$ and $\mathrm{GABA}_{\mathrm{B}} \mathrm{Rs}^{36}{ }^{36}$ It was found that adult rat DRG neurons preferentially contain $\alpha 2 \beta 3 \gamma 2$ subunits of $\mathrm{GABA}_{\mathrm{A}} \mathrm{R} \cdot{ }^{37} \mathrm{As}$ it is well known, the $\mathrm{GABA}_{\mathrm{A}} \mathrm{R}$ belongs to the superfamily of ligand-gated channel receptor; thus, if the $\mathrm{Cl}-$ channel is opened, $\mathrm{GABA}_{\mathrm{A}} \mathrm{R}$ promotes primary afferent depolarization, resulting in a decrease in release of neurotransmitters including SP from the primary afferent terminals. ${ }^{8}$ Furthermore, the $\mathrm{Na}-\mathrm{K}-2 \mathrm{Cl}$ cotransporters (NKCC), expressed in virtually all DRG neurons in the rat, accumulate chloride ions intracellularly ${ }^{38}$ and could mediate an enhanced primary afferent 
A $\mathrm{GABA}_{\mathrm{A}} \alpha 2 \mathrm{R}$ mRNA
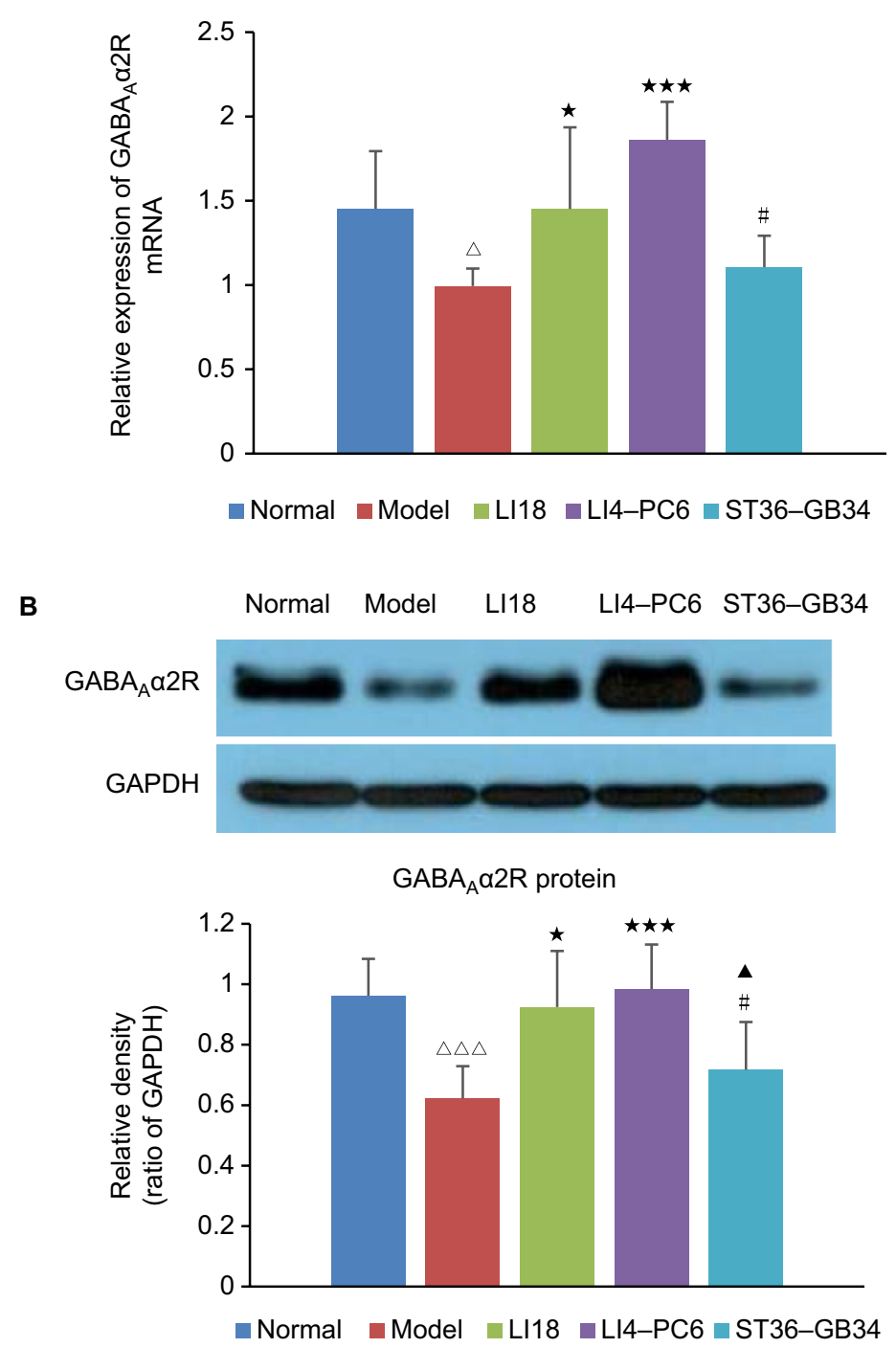

Figure 5 Effect of EA on expression of GABA $_{A} \alpha 2 R$ mRNA and protein in C3-6 DRGs of incisional neck pain rats.

Notes: (A) The mRNA expression of GABA $A_{A} \alpha 2 R$ and $(B)$ the protein expression of $G A B A_{A} \alpha 2 R$. Expression of GABA $\alpha 2 R$ mRNA and protein was decreased in the model group in comparison with the normal group. EA at LII8 and LI4-PC6 could upregulate the expression of GABA $\alpha 2 R$ mRNA and protein in C3-6 DRGs compared with the model group ( $n=5$ in each group). Data were analyzed using one-way ANOVA and expressed as mean \pm SD of the mean. ${ }^{\triangle P}<0.05, \Delta \Delta \Delta P<0.001$ vs the normal group; ${ }^{*} P<0.05$, $* * * P<0.00$ I vs the model group; ${ }^{\wedge} P<0.05$ vs the LII 8 group; ${ }^{*} P<0.05$ vs the LI4-PC6 group.

Abbreviations: DRG, dorsal root ganglion; EA, electroacupuncture; GABA, $\gamma$-aminobutyric acid.

depolarization. ${ }^{39}$ Being identical to the result of an absence of GABA depolarization in DRG neurons, the $\mathrm{NKCC}-/-$ mice showed lower sensitivity to thermal stimulus (longer paw withdrawal latency), ${ }^{40}$ indicating that the NKCC participates in the modulation of GABA neurotransmission and sensory perception. A previous study revealed that NKCC1 mRNA, colocalized with CGRP and transient receptor potential cation channel subfamily V member 1 (TRPV1), is restricted to the small and medium diameter sensory neurons of DRGs and trigeminal ganglions in adult rats, and $\mathrm{NKCC} 1$ plays a role in maintaining high intracellular chloride levels in peptidergic and nociceptive neurons, respectively. ${ }^{41}$ The facilitation or inhibition of nociception transmitting to the spinal cord depended, to some degree, on the interaction between the GABAergic modulation and the peptidergic nociceptive transmission in the DRGs. Since the activation of GABA $A_{A}$ in sensory DRG neurons induces presynaptic inhibition at the level of the spinal $\mathrm{DH},{ }^{42}$ if GABA responses were depressed, the disinhibition of the presynaptic inhibition would result in facilitation of nociceptive information transmission in the spinal cord. ${ }^{8}$ Our previous study showed that at 48 hours after operation, the immunoactivity and mRNA expression 

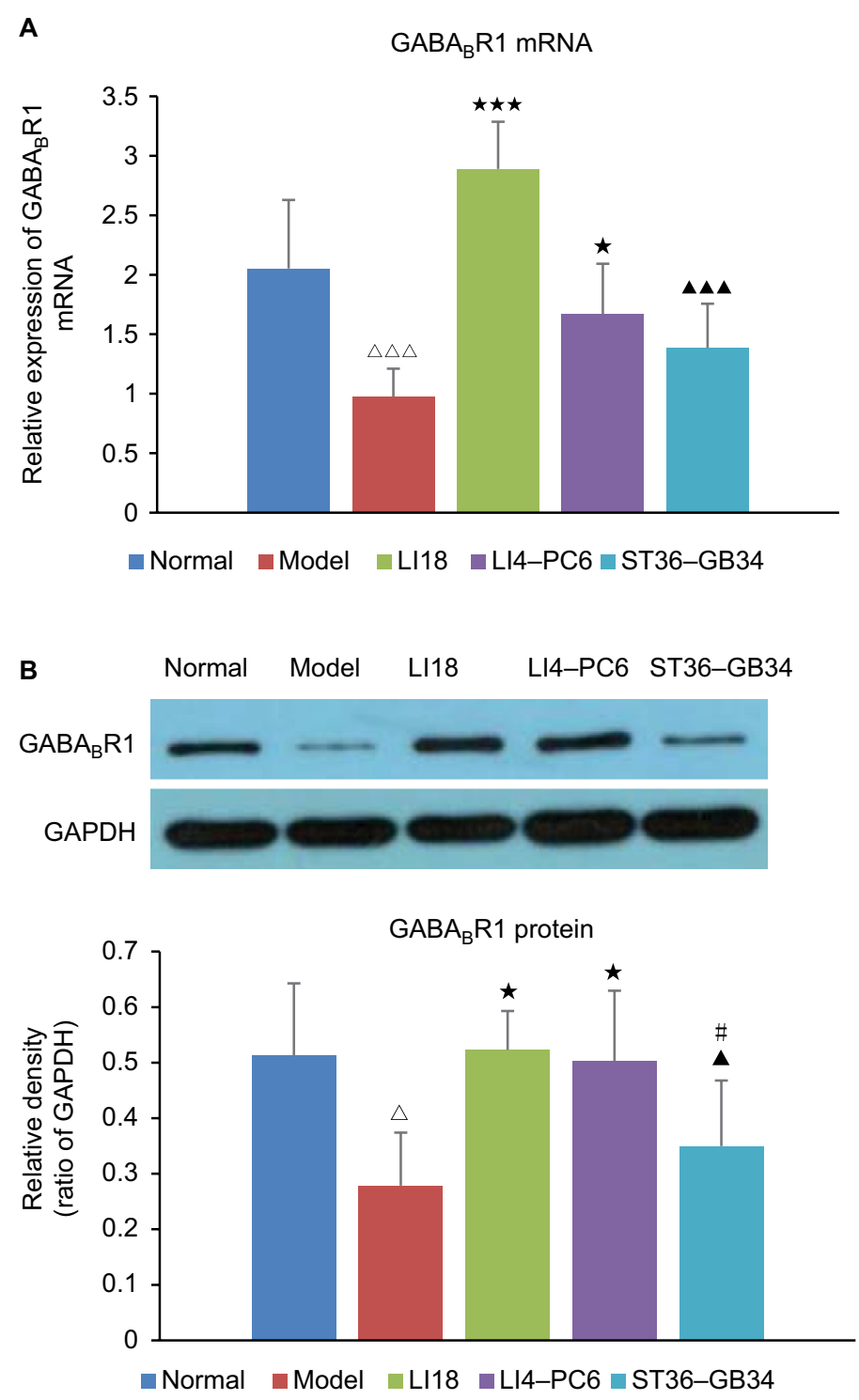

Figure 6 Effect of EA on the expression of $G A B A_{B} R I$ mRNA and protein in C3-6 DRGs of incisional neck pain rats.

Notes: (A) The mRNA expression of $G A B A_{B} R I$. (B) Protein expression of $G A B A_{B} R I$. Expression of $G A B A_{B} R I$ mRNA and protein was decreased in the model group in comparison with the normal group. EA at LII8 and LI4-PC6 could upregulate the expression of GABA ${ }_{B} R I$ mRNA and protein in C3-6 DRGs ( $n=5$ per group). Data were analyzed using one-way ANOVA and expressed as mean \pm SD of the mean. ${ }^{\triangle} P<0.05, \triangle \triangle \Delta P<0.001$ vs the normal group; ${ }^{*} P<0.05$, ${ }^{*} * * P<0.001$ vs the model group; ${ }^{\wedge} P<0.05$, $\triangle \triangle P<0.001$ vs the LII8 group; ${ }^{P}<0.05$ vs the LI4-PC6 group.

Abbreviations: DRG, dorsal root ganglion; EA, electroacupuncture; GABA, $\gamma$-aminobutyric acid.

of SP and CGRP were significantly increased along with a decrease of thermal PT. ${ }^{25}$ In the present study, we further found that after cervical incision, the immunoactivity, gene and protein expression of $\mathrm{GABA}_{\mathrm{A}} \alpha 2 \mathrm{R}$ were downregulated accompanied with upregulation of SP immunoactivity, which means an interaction occurred between $\mathrm{GABA}_{\mathrm{A}} \mathrm{R}$ and $\mathrm{SP}$. Similar results were reported in neuropathic pain rats. ${ }^{43}$ While $\mathrm{GABA}_{\mathrm{A}}$ receptor agonists applied to the $\mathrm{L} 5 \mathrm{DRG}$ at the time of a sciatic nerve crush injury caused dose-dependent, longlasting alleviation of thermal hyperalgesia, $\mathrm{GABA}_{\mathrm{A}}$ receptor antagonists, bicuculline and picrotoxin, exacerbated thermal hyperalgesia, ${ }^{44}$ indicating an important role of GABAergic modulation in DRGs.

In regard to the action of SGCs in DRGs, it has been demonstrated that they play a crucial role in nociceptive signal transmission. ${ }^{17,45} \mathrm{SGCs}$ have marked $\mathrm{K}^{+}$permeability to maintain normal extracellular $\mathrm{K}^{+}$concentrations, being closely associated with altered excitability of primary sensory neurons. ${ }^{46} \mathrm{The}_{\mathrm{GABA}} \mathrm{R}$, a $\mathrm{G}$ protein-coupled receptor, consists of two subunits, $\mathrm{GABA}_{\mathrm{B}} 1$ and $\mathrm{GABA}_{\mathrm{B}} 2{ }^{47} \mathrm{SGCs}$ 
were found to be labeled with $\mathrm{GABA}_{B} \mathrm{R}$ antibody. ${ }^{21} \mathrm{GABA}_{B} \mathrm{R}$ could activate potassium ion channels (Kir) via $G$ protein $\beta \gamma$ subunits, ${ }^{48}$ and the Kir channels function in establishing a high potassium conductance selectivity of the glial cell membrane and a strongly negative resting potential. It was reported that the peripheral inflammatory impairment of glial potassium homeostasis in the sensory ganglia suppressing Kir4.1 currents contributes to trigeminal inflammatory pain. ${ }^{49}$ By using immunohistochemical techniques, it was demonstrated that $\mathrm{GABA}_{\mathrm{B}} \mathrm{R}$ was coexpressed with SGCs in the trigeminal ganglions. $\mathrm{GABA}_{\mathrm{B}} \mathrm{R}$ activation could potentiate the Kir current in SGCs, and the released GABA from the TRG neuronal soma could contribute to buffering of extracellular potassium ion $\left(\mathrm{K}^{+}\right)$concentrations following excitation of TRG neurons during the processing of sensory information, including the transmission of pain signals. ${ }^{50}$ This buffering activity took up $\mathrm{K}^{+}$to offset extracellular $\mathrm{K}^{+}$ accumulation during neuronal firing, ${ }^{51}$ thus preventing action potential "short-circuiting" and uncontrolled excitability changes. ${ }^{52}$ Our previous study showed that cervical incision could increase the immunoactivity and mRNA expression of GFAP and upregulate the mRNA expression and protein content of proinflammatory cytokines IL-1 $\beta$, IL-6, and tumor necrosis factor- $\alpha$ in $\mathrm{C} 3-6 \mathrm{DRGs},{ }^{26}$ suggesting that the activation of SGCs and subsequent release of proinflammatory cytokines played an important role in maintaining incisional pain. In the present experiment, immunofluorescence double labeling of C3-6 DRG tissues displayed that SP-IR-positive cells were small-sized neurons, GABA $\alpha 2 R$ IR-positive cells were small- and medium-sized neurons, while $\mathrm{GABA}_{B} \mathrm{R} 1 \mathrm{IR}$ positive cells belonged to SGCs. These results are basically identical to Poorkhalkali et al's findings that DRG neurons were positive for both the $G_{A B A} R 1$ and $G_{A B A} R 1$ antibodies, but the former stained the cells much more intensely. Satellite cells were labeled with the $\mathrm{GABA}_{\mathrm{B}} \mathrm{R} 1$ antibody. ${ }^{21}$ Thus, we speculate that EA at LI18 and LI4-PC6 possibly enhanced the GABA released from C3-6 DRG neurons which bind $\mathrm{GABAB}$ receptor $\mathrm{G} \beta \gamma$ subunit to activate Kir4.1 current in the SGCs, further decreasing the extracellular $\mathrm{K}^{+}$concentration to inactivate the SGCs, Consequently, the release of proinflammatory cytokines was reduced and the nociceptive signal transmission to the spinal DH was suppressed, resulting in pain relief at last. However, the detailed processing course needs to be studied further.

As for the selection of stimulating frequency $2 / 100 \mathrm{~Hz}$ of EA in the present study, we mainly consider the following: 1) keeping the efficacy of EA stimulation by avoiding possible adaptation or tolerance of continuous electrical stimulation in the rat if one fixed frequency is used and 2) spinal cord GABA has been demonstrated to be involved in the antinociceptive effect of EA stimulation of ST36 and Sanyinjiao (SP6) at both 2 and 2/100 Hz in hind paw incisional pain rats. ${ }^{53}$ Our findings of the present study that GABAergic modulation in the C3-6 DRGs was implicated in the analgesic effect of EA at $2 / 100 \mathrm{~Hz}$ by way of enhancing the function of GABARs and subsequently promoting the interaction between $\mathrm{SP}+$ and GABA+ neurons and between GABA+ neurons and SGCs in cervical DRGs in incisional neck pain rats are thus highly reasonable. But the limitation of this study is lack of detailed interaction process, which we will investigate further.

\section{Conclusion}

EA stimulation of LI18 and LI4-PC6 has an analgesic effect in incisional neck pain rats, which is closely associated with its effects in upregulating the levels of $\mathrm{GABA}_{\mathrm{A}} \mathrm{R}$ expressing on SP neurons and $\mathrm{GABA}_{B} \mathrm{R}$ expressing on SGCs and downregulating the activities of SP neurons and SGCs in C3-6 DRGs. These data suggest that interactions between neurons and between neurons and SGCs may contribute to the analgesic effect of EA of LI18 and LI4PC6. These results provide an experimental evidence for application of EA therapy for relieving postoperative pain of thyroidectomy.

\section{Acknowledgments}

This study was supported by the Youth Project of National Natural Science Foundation of China grant (No. 81303055), "973" program of the Ministry of Science and Technology of China (Nos. 2014CB543103; 2013CB531904) and the independent project of Institute of Acupuncture and Moxibustion (ZZ201711006). We would like to thank Dr Wan-Zhu Bai for the technical support in confocal images capture and analysis.

\section{Author contributions}

Li Na Qiao, Yong Sheng Yang and Jun Ling Liu contributed equally to this work. Li Na Qiao performed the experiments and manuscript drafting and revising. Yong Sheng Yang and Jun Ling Liu designed this study and revised the manuscript. Jiang Zhu gave valuable advice about the experimental design. Lian Hong Tan and Yi Nan Shi assisted with the experimental work and data collection. Bing Zhu and Pei Jing Rong supervised the experiments and agreed to be accountable for all aspects of the work. All authors contributed to data analysis, drafting or revising the article, gave final approval of the version to be published, and agree to be accountable for all aspects of the work. 


\section{Disclosure}

The authors report no conflicts of interest in this work.

\section{References}

1. Mitra S, Carlyle D, Kodumudi G, Kodumudi V, Vadivelu N. New advances in acute postoperative pain management. Curr Pain Headache Rep. 2018;22(5):35.

2. Liem L. Stimulation of the dorsal root ganglion. Prog Neurol Surg. 2015;29:213-224.

3. Krames ES. The dorsal root ganglion in chronic pain and as a target for neuromodulation: a review. Neuromodulation. 2015;18(1):24-32.

4. Eldabe S, Burger K, Moser H, et al. Dorsal root ganglion (DRG) stimulation in the treatment of phantom limb pain (PLP). Neuromodulation. 2015;18(7):610-617.

5. Pan B, Zhang Z, Chao D, Hogan QH. Dorsal root ganglion field stimulation prevents inflammation and joint damage in a rat model of rheumatoid arthritis. Neuromodulation. 2018;21(3):247-253.

6. Mccarthy PW, Lawson SN. Cell type and conduction velocity of rat primary sensory neurons with substance P-like immunoreactivity. Neuroscience. 1989;28(3):745-753.

7. Si JQ, Li ZW. Effect of substance P on the somatic membrane of rat DRG neurons. Sheng Li Xue Bao. 1996;48(1):8-14.

8. Si JQ, Zhang ZQ, Li CX, Wang LF, Yang YL, Li ZW. Modulatory effect of substance $\mathrm{P}$ on GABA-activated currents from rat dorsal root ganglion. Acta Pharmacol Sin. 2004;25(5):623-629.

9. Lin Q, Peng YB, Willis WD. Role of GABA receptor subtypes in inhibition of primate spinothalamic tract neurons: difference between spinal and periaqueductal gray inhibition. $J$ Neurophysiol. 1996;75(1):109-123.

10. Malcangio M, Bowery NG. GABA and its receptors in the spinal cord. Trends Pharmacol Sci. 1996;17(12):457-462.

11. Toulmé E, Blais D, Léger C, et al. An intracellular motif of P2X(3) receptors is required for functional cross-talk with GABA(A) receptors in nociceptive DRG neurons. $J$ Neurochem. 2007;102(4): 357-1368.

12. Labrakakis C, Tong CK, Weissman T, Torsney C, Macdermott AB. Localization and function of ATP and GABAA receptors expressed by nociceptors and other postnatal sensory neurons in rat. $J$ Physiol. 2003;549(Pt 1):131-142.

13. Hu HZ, Li ZW, Si JQ. Evidence for the existence of substance $P$ autoreceptor in the membrane of rat dorsal root ganglion neurons. Neuroscience. 1997;77(2):535-541.

14. Ma KT, Si JQ, Zhang ZQ, et al. Modulatory effect of CCK-8S on GABA-induced depolarization from rat dorsal root ganglion. Brain Res. 2006;1121(1):66-75.

15. Li L, Zhao L, Wang Y, et al. PKCe mediates substance P inhibition of GABAA receptors-mediated current in rat dorsal root ganglion. J Huazhong Univ Sci Technolog Med Sci. 2015;35(1):1-9.

16. Pannese E. The structure of the perineuronal sheath of satellite glial cells (SGCs) in sensory ganglia. Neuron Glia Biol. 2010;6(1):3-10.

17. Liu FY, Sun YN, Wang FT, et al. Activation of satellite glial cells in lumbar dorsal root ganglia contributes to neuropathic pain after spinal nerve ligation. Brain Res. 2012;1427:65-77.

18. Miyagi M, Ohtori S, Ishikawa T, et al. Up-regulation of TNFalpha in DRG satellite cells following lumbar facet joint injury in rats. Eur Spine J. 2006;15(6):953-958.

19. Dev R, Srivastava PK, Iyer JP, Dastidar SG, Ray A. Therapeutic potential of matrix metalloprotease inhibitors in neuropathic pain. Expert Opin Investig Drugs. 2010;19(4):455-468.

20. Takeda M, Tanimoto T, Kadoi J, et al. Enhanced excitability of nociceptive trigeminal ganglion neurons by satellite glial cytokine following peripheral inflammation. Pain. 2007;129(1-2):155-166.

21. Poorkhalkali N, Juneblad K, Jönsson AC, et al. Immunocytochemical distribution of the GABA(B) receptor splice variants GABA(B) R1a and R1b in the rat CNS and dorsal root ganglia. Anat Embryol (Berl). 2000;201(1):1-13.
22. Han JS. Acupuncture analgesia: areas of consensus and controversy. Pain. 2011;152(3 Suppl):S41-S48.

23. Tu WZ, Cheng RD, Cheng B, et al. Analgesic effect of electroacupuncture on chronic neuropathic pain mediated by $\mathrm{P} 2 \mathrm{X} 3$ receptors in rat dorsal root ganglion neurons. Neurochem Int. 2012;60(4):379-386.

24. Wang L, Zhang Y, Dai J, Yang J, Gang S. Electroacupuncture (EA) modulates the expression of NMDA receptors in primary sensory neurons in relation to hyperalgesia in rats. Brain Res. 2006;1120(1):46-53.

25. Qiao LN, Liu JL, Tan LH, Yang HL, Zhai X, Yang YS. Effect of electroacupuncture on thermal pain threshold and expression of calcitonin-gene related peptide, substance $\mathrm{P}$ and $\gamma$-aminobutyric acid in the cervical dorsal root ganglion of rats with incisional neck pain. Acupunct Med. 2017;35(4):276-283.

26. Qiao LN, Tan LH, Yang JJ, et al. Effects of electroacupuncture on activities of satellite glial cells of dorsal root ganglia in rats with neck incision pain. Zhen Ci Yan Jiu. 2017;42(4):283-289.

27. Liu Y, Ni Y, Zhang W, Sun YE, Ma Z, Gu X. Antinociceptive effects of caloric restriction on post-incisional pain in nonobese rats. Sci Rep. 2017;7(1):1805.

28. Li ZR. Experimental Acupuncturolog. Beijing, China: Traditional Chinese Medicine Publishing House; 2012:255-256.

29. Silva JR, Silva ML, Prado WA. Analgesia induced by $2-$ or $100-H z$ electroacupuncture in the rat tail-flick test depends on the activation of different descending pain inhibitory mechanisms. J Pain. 2011;12(1):51-60.

30. Takeda M, Tanimoto T, Nasu M, Ikeda M, Kadoi J, Matsumoto S. Activation of NK1 receptor of trigeminal root ganglion via substance $P$ paracrine mechanism contributes to the mechanical allodynia in the temporomandibular joint inflammation in rats. Pain. 2005;116(3):375-385

31. Li J, Vause CV, Durham PL. Calcitonin gene-related peptide stimulation of nitric oxide synthesis and release from trigeminal ganglion glial cells. Brain Res. 2008;1196:22-32.

32. Kung LH, Gong K, Adedoyin M, et al. Evidence for glutamate as a neuroglial transmitter within sensory ganglia. PLoS One. 2013;8(7):e68312.

33. Zhang X, Chen Y, Wang C, Huang LY. Neuronal somatic ATP release triggers neuron-satellite glial cell communication in dorsal root ganglia. Proc Natl Acad Sci U S A. 2007;104(23):9864-9869.

34. Hayasaki H, Sohma Y, Kanbara K, Maemura K, Kubota T, Watanabe M. A local GABAergic system within rat trigeminal ganglion cells. Eur J Neurosci. 2006;23(3):745-757.

35. Zubrzycka M, Janecka A. Substance P: transmitter of nociception (Minireview). Endocr Regul. 2000;34(4):195-201.

36. Salio C, Merighi A, Bardoni R. GABAB receptors-mediated tonic inhibition of glutamate release from $A \beta$ fibers in rat laminae III/IV of the spinal cord dorsal horn. Mol Pain. 2017;13:174480691771004.

37. Ma W, Saunders PA, Somogyi R, Poulter MO, Barker JL. Ontogeny of GABAA receptor subunit mRNAs in rat spinal cord and dorsal root ganglia. J Comp Neurol. 1993;338(3):337-359.

38. Alvarez-Leefmans FJ, León-Olea M, Mendoza-Sotelo J, Alvarez FJ, Antón B, Garduño R. Immunolocalization of the $\mathrm{Na}(+)-\mathrm{K}(+)-2 \mathrm{Cl}(-)$ cotransporter in peripheral nervous tissue of vertebrates. Neuroscience. 2001;104(2):569-582.

39. Price TJ, Cervero F, de Koninck Y. Role of cation-chloride-cotransporters (CCC) in pain and hyperalgesia. Curr Top Med Chem. 2005;5(6):547-555.

40. Sung KW, Kirby M, Mcdonald MP, Lovinger DM, Delpire E. Abnormal GABAA receptor-mediated currents in dorsal root ganglion neurons isolated from $\mathrm{Na}-\mathrm{K}-2 \mathrm{Cl}$ cotransporter null mice. J Neurosci. 2000;20(20):7531-7538.

41. Price TJ, Hargreaves KM, Cervero F. Protein expression and mRNA cellular distribution of the NKCC1 cotransporter in the dorsal root and trigeminal ganglia of the rat. Brain Res. 2006;1112(1):146-158.

42. Sluka KA, Willis WD, Westlund KN. Inflammation-induced release of excitatory amino acids is prevented by spinal administration of a GABAA but not by a GABAB receptor antagonist in rats. $J$ Pharmacol Exp Ther. 1994;271(1):76-82.

43. Obata K, Yamanaka H, Fukuoka T, et al. Contribution of injured and uninjured dorsal root ganglion neurons to pain behavior and the changes in gene expression following chronic constriction injury of the sciatic nerve in rats. Pain. 2003;101(1-2):65-77. 
44. Naik AK, Pathirathna S, Jevtovic-Todorovic V. GABAA receptor modulation in dorsal root ganglia in vivo affects chronic pain after nerve injury. Neuroscience. 2008;154(4):1539-1553.

45. Takeda M, Takahashi M, Matsumoto S. Contribution of the activation of satellite glia in sensory ganglia to pathological pain. Neurosci Biobehav Rev. 2009;33(6):784-792.

46. Laming PR, Kimelberg H, Robinson S, et al. Neuronal-glial interactions and behaviour. Neurosci Biobehav Rev. 2000;24(3):295-340.

47. Laffray S, Bouali-Benazzouz R, Papon MA, et al. Impairment of GABAB receptor dimer by endogenous $14-3-3 \zeta$ in chronic pain conditions. EMBO J. 2012;31(15):3239-3251.

48. Turecek R, Schwenk J, Fritzius T, et al. Auxiliary GABAB receptor subunits uncouple $G$ protein $\beta \gamma$ subunits from effector channels to induce desensitization. Neuron. 2014;82(5):1032-1044.

49. Takeda M, Takahashi M, Nasu M, Matsumoto S. Peripheral inflammation suppresses inward rectifying potassium currents of satellite glial cells in the trigeminal ganglia. Pain. 2011;152(9):2147-2156.
50. Takeda M, Nasu M, Kanazawa T, Shimazu Y. Activation of GABA(B) receptors potentiates inward rectifying potassium currents in satellite glial cells from rat trigeminal ganglia: in vivo patch-clamp analysis. Neuroscience. 2015;288:51-58.

51. D'Ambrosio R, Gordon DS, Winn HR. Differential role of KIR channel and $\mathrm{Na}(+) / \mathrm{K}(+)$-pump in the regulation of extracellular $\mathrm{K}(+)$ in rat hippocampus. J Neurophysiol. 2002;87(1):87-102.

52. Tsantoulas $\mathrm{C}$, Mcmahon SB. Opening paths to novel analgesics: the role of potassium channels in chronic pain. Trends Neurosci. 2014;37(3):146-158

53. da Silva JR, da Silva ML, Prado WA. Electroacupuncture at $2 / 100 \mathrm{~Hz}$ activates antinociceptive spinal mechanisms different from those activated by electroacupuncture at 2 and $100 \mathrm{~Hz}$ in responder rats. Evid Based Complement Alternat Med. 2013;2013:1-14.

\section{Journal of Pain Research}

\section{Publish your work in this journal}

The Journal of Pain Research is an international, peer reviewed, open access, online journal that welcomes laboratory and clinical findings in the fields of pain research and the prevention and management of pain. Original research, reviews, symposium reports, hypothesis formation and commentaries are all considered for publication.

\section{Dovepress}

The manuscript management system is completely online and includes a very quick and fair peer-review system, which is all easy to use. Visit http://www.dovepress.com/testimonials.php to read real quotes from published authors. 